\title{
L'HOMME L'Homme
}

165 | janvier-mars 2003

Image et anthropologie

\section{De l'archaïque au commencement}

La pensée du dessin chez Joseph Beuys

Jean-Philippe Antoine

\section{(2) OpenEdition}

Journals

\section{Édition électronique}

URL : http://journals.openedition.org//homme/200

DOI : $10.4000 /$ Ihomme.200

ISSN : 1953-8103

Éditeur

Éditions de l'EHESS

\section{Édition imprimée}

Date de publication : 1 janvier 2003

Pagination : 129-142

ISBN : 2-7132-1779-2

ISSN : 0439-4216

Référence électronique

Jean-Philippe Antoine, " De l'archaïque au commencement », L'Homme [En ligne], 165 | janvier-mars 2003, mis en ligne le 27 mars 2008, consulté le 03 mai 2019. URL : http://journals.openedition.org/ Ihomme/200; DOI : 10.4000/lhomme.200 


\title{
De l'archaïque au commencement \\ La pensée du dessin chez Joseph Beuys
}

\author{
Jean-Philippe Antoine
}

\begin{abstract}
Q UI NE SAIT rien de son œuvre, connaît pourtant le slogan par lequel l'artiste allemand Joseph Beuys (1921-1986) s'est rendu célèbre : "Chaque être humain est un artiste ». Peut-être connaîtra-t-on même l'expression a priori mystérieuse de "concept élargi de l'art». Le slogan fait valoir que tout être humain est capable d'actes de création. En conséquence la société a la vocation - et le devoir - de favoriser cette disposition, sous peine de susciter ou de perpétuer des maux et souffrances qui l'affectent dans sa totalité et qui remettent en cause son intégrité. Quant à l'expression de " concept élargi de l'art", sans laquelle le slogan reste ouvert à tous les malentendus, elle déclare que l'art n'est pas l'activité séparée, minoritaire et rare que les sociétés bourgeoises ont confiée à des artistes spécialisés, cantonnés pour l'essentiel dans la production de peintures et sculptures à visée décorative. Il est bien plutôt l'activité par laquelle les hommes, au niveau des individus comme des divers groupes sociaux, se sculptent eux-mêmes, c'està-dire construisent leurs pensées, leur mémoire et leur histoire au travers des institutions qui gouvernent et organisent leurs vies. Cette activité, qui se confond avec l'esthétique, a valeur " anthropologique ${ }^{1}$.

En revendiquant pour l'art un statut aussi fondamental, Beuys condamne à l'évidence les "Beaux-arts" tels qu'ils ont été entendus depuis plusieurs siècles. Il condamne également une partie non négligeable des avant-gardes du XXe siècle, dont le désir révolutionnaire est en pratique resté fondé sur la définition « étroite » de l'art qu'elles s'étaient vouées à remettre en question. Certes, le risque assumé par la position beuysienne est, à force de dissoudre les concepts traditionnels de l'art, d'aboutir à une définition tellement ample qu'elle échoue à rendre compte, d'une part de la spécificité des activités spécialisées nommées art dans les sociétés occidentales depuis quelques siècles, d'autre part de la nature
\end{abstract}

1. Voir par exemple, Irmeline Lebeer, "Entretien avec Joseph Beuys ", Cahiers du Musée national d'Art moderne, 1980, 4 : 171-193. 
multiple des activités "créatives" de construction sociale auxquelles s'adonnent les diverses sociétés humaines. Le "concept élargi de l'art» est-il autre chose qu'une coquille vide? La revendication de la qualification d'artiste rend-elle compte d'une composante nécessaire de toute activité humaine? L'examen du statut du dessin - sans doute l'activité la plus fondamentale que pratique l'artiste dans tout le cours de son ouvre - permet de montrer comment la critique beuysienne de l'art en tant qu'activité spécialisée et raréfiée, ne se résout pas dans une condamnation des activités et des produits établis sous son étiquette. Elle permet au contraire à ceux-ci de trouver une place inédite au côté des productions humaines qu'ils avaient jusque-là servi à exclure ou à minorer. Dans ce concept déhiérarchisé de l'art résident des possibilités artistiques et démocratiques inédites, qui jettent les bases d'une pensée anthropologique des images débarrassée des préjugés occidentalistes et primitivistes.

\section{La réalité du dessin}

L'élargissement de la catégorie du dessin se manifeste d'abord chez Beuys par la diversité des modèles de sa propre pratique. Ils embrassent l'entier territoire de l'art, des productions préhistoriques ou primitives jusqu'à la modernité, et expriment le refus de s'attacher à un style. Comme l'a écrit Werner Hofmann :

"Avant que le dessin, aux alentours de 1400, ne s'autonomise en tant que document digne de conservation d'une recherche de forme subjective, les moyens graphicolinéaires avaient un champ de manœuvres par rapport auquel le genre "dessin" signifie une perte de terrain. Le dessinateur Beuys exige de quiconque a affaire à lui, qu'il reconnaisse cela. Le regard doit retourner vers les dessins des cavernes et les gravures sur os préhistoriques, il doit embrasser les productions ornementales et les initiales de l'enluminure médiévale, et la précision des tracés architecturaux gothiques, mais aussi les gribouillis sous-esthétiques et sous-culturels et les graffiti anonymes, les arabesques et les hiéroglyphes de toute sorte. $»^{2}$

Le premier geste de Beuys consiste en effet à reconsidérer l'histoire occidentale du dessin à partir d'un point de vue précédant sa scission en dessin scientifique et artistique :

"On peut dire que le dessin, depuis la Renaissance, suit deux voies parallèles. Celle du dessin scientifique qui s'étend de plus en plus, allant jusqu'aux diagrammes, formulessignes, schémas de physique, coupes techniques. Celle du dessin artistique qui est ancré dans de tout autres lieux, relevant pour ainsi dire d'intérêts bien différents pour la connaissance philosophique, psychologique, allégorique ou symbolique. Ces deux courants travaillent sur un plan plus élémentaire à la métamorphose de la conscience humaine. Le rôle du dessin est ancien. Les peintures rupestres, les figurations sur le sable, le tracé de lignes sur le sol, tel qu'on le rencontre dans les cultures indiennes, c'est en principe du dessin. Le contour pur, tel qu'il apparaît dans les premiers dessins ou le dessin compris comme signe (croix, cercle ou ligne en zig-zag), chacun peut pra-

2. W. Hofmann, «Die innere Form ", in Beuys vor Beuys, Cologne, Dumont, $1987: 33$. 


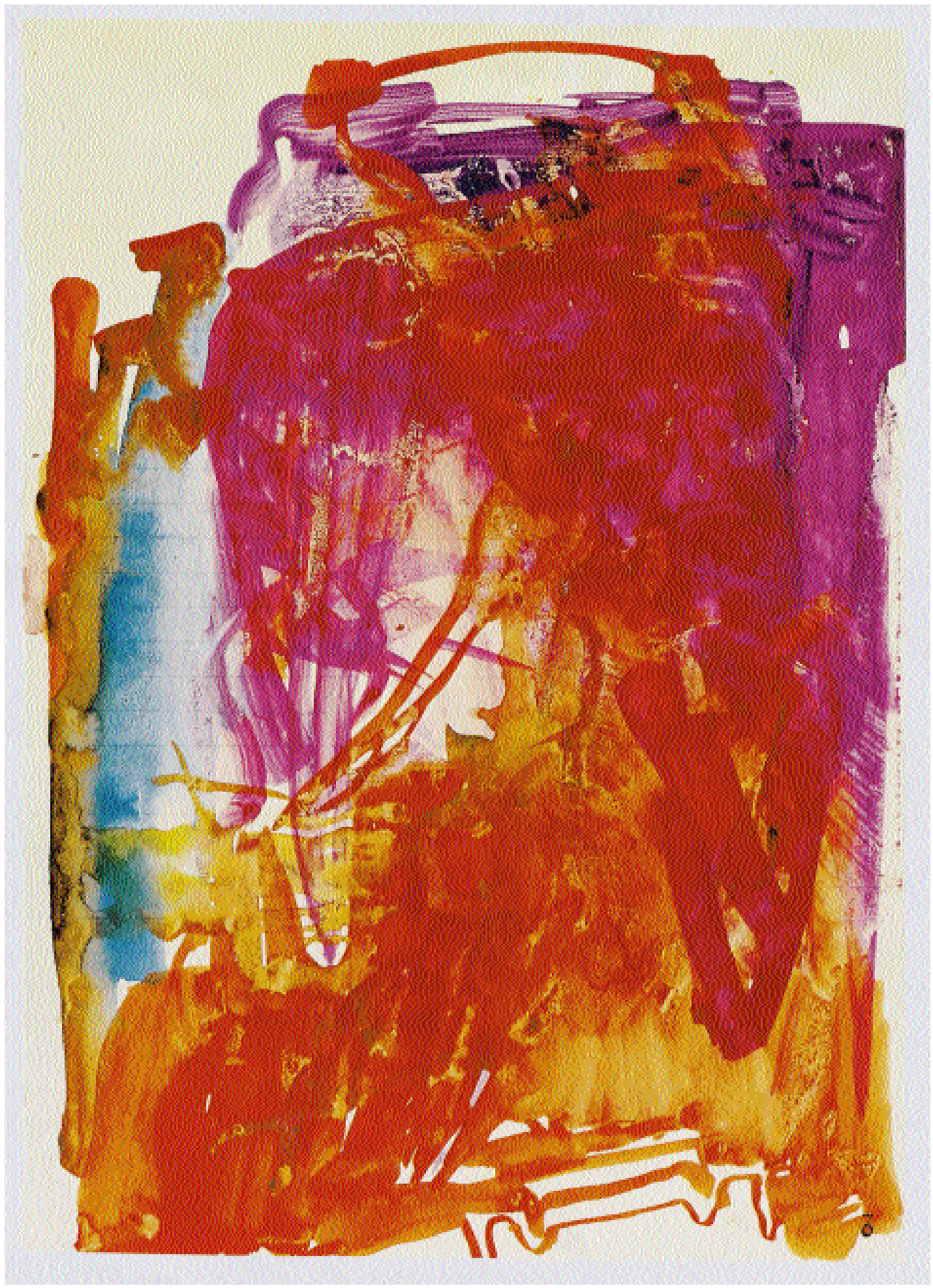

Fig. I Accumulator (Enthladung), 1959, gouache, $29,5 \times 21,4 \mathrm{~cm}$

(Joseph Beuys, Natur, Materie, Form, Munich, Schirmer-Mosel, I991: pl. I59) 
tiquement le modifier à l'infini. Tout cela offre différents aspects des possibilités d'expression du dessin, sans qu'il soit nécessaire de développer un style propre. » ${ }^{3}$

En réintégrant le dessin scientifique dans la tradition occidentale du dessin, généralement pensée soit sur le mode de la considération exclusive du dessinartistique, soit sur celui de la séparation des deux genres en entités absolument distinctes, et hiérarchiquement déterminées, Beuys rend possible de penser leur origine commune. Cette origine déborde résolument les frontières du métier artistique, tel que le définit l'histoire de l' a rt (qu'il soit celui de "l'âge de la représentation » ou encore, depuis la fin du XIXe siècle, celui de productions autres - "primitives", archaïques ou exotiques). Elle rend par là même caduque la notion de style.

La manière beuysienne de dessiner - le mot de manière désignant ici justement autre chose que la notion de style - se distingue alors à la fois par le vaste réservoir de formes auquel elle fait appel, et par le refus de cantonner le geste accompli dans un genre préétabli, aisément reconnaissable. Ces dessins provoquent souvent une première réaction d'indécision, voire d'agacement, du spectateur: à quoi a-t-il affaire exactement? À quel(s) registre(s) appartiennent les signes et traces qui occupent la feuille de papier ou de carton placée devant ses yeux ? Taches, accidents (Fig. 1), esquisses de traits inachevés, formes en gestation (Fig. 2), mais aussi diagrammes d'allure technique ou scientifique (Fig. 3), le tout parfois présent sur un même feuillet, juxtaposé ou superposé. Dans la multiplicité des types de traces présentés, dans l'indécision quant à leur statut suscitée par l'absence (revendiquée) d'un style dominant, réside un effet non négligeable de dépaysement et de remise en question des hiérarchies. Le regard apprend à naviguer entre des modes d'expression qu'il considérait jusque-là comme incompatibles, ou qu'il n' a vait jamais vus rassemblés. Il appréhende ainsi non seulement leur compossibilité mais ce qui la fonde : apparaissent en effet dans ce lieu inédit des caractères propres à tout type de dessin. Ces caractères ne relèvent pas d'une appréhension formelle des résultats, mais de l'activité même mise en jeu dans le dessin.

Ce que pratiquent et révèlent les œuvres de Beuys est en effet une conception du dessin qui embrasse toute forme de trace ou de marque gestuelle faite par l'homme sur les supports les plus divers (papier mais aussi murs, sable, roche). Elle a pour critère fondamental la capacité du geste à déclarer un ensemble de relations, une ressemblance au sens large, quelqu'en soit le registre :

«Il n’y a rien de plus élémentaire que le dessin. Quand je montre à quelqu'un le chemin et que je lui indique sur un bout de papier le tracé des rues, eh bien je dessine. Au fond dessiner, n'est-ce pas, ce n'est rien d'autre que faire un plan, ou visualiser quelque chose, un ensemble de relations spatiales ou tout simplement un rapport de grandeurs. [...] On ne doit jamais dire : celui-là il sait dessiner, et moi je ne sais pas. Ce n'est pas du tout ça. Tout homme peut dessiner, bien sûr qu'il peut dessiner, tant qu'il a des mains. Et même s'il n'a pas de mains, il peut dessiner avec les pieds. $»^{4}$

3. Hans van der Grinten-Joseph Beuys, "Dialogue », in Max Reithmann, Joseph Beuys. La mort me tient en éveil, Toulouse, Arpap, $1994: 22$.

4. Joseph Beuys \& Volker Harlan, Qu'est-ce que l'art?, traduit par Laurent Cassagnau. Paris, L'Arche, $1992: 49$. 
Il n'est pas jusqu'au geste d'écrire qui ne rentre lui aussi dans la catégorie du dessin :

"Nous avons parlé de la parole, écrire par exemple, c'est aussi dessiner. Si on regarde juste un peu ce que fait la main, ces drôles de formes, c'est aussi du dessin. C'est pourquoi je dis que tout homme est un dessinateur en ce sens qu'il représente quelque chose, tout homme représente, les uns davantage, les autres moins ; cela dépend bien sûr de la décision qu'on a prise pour son métier. Mais le dessin apparaît de toute façon dans la vie consciente. $\|^{5}$

Cessant d'être défini par l'emploi de techniques et de matériaux particuliers, le dessin au sens élargi caractérise tout type de déplacement le long de la ligne de crête qui relie, à chaque fois sous forme d'un événement singulier, un geste de dénotation et son enregistrement comme tel dans une empreinte matérielle susceptible de durée. Prélèvement "épiphanique ", au sens joycien ${ }^{6}$, dans le continuum du réel, cet acte ne possède aucune forme préassignée, pas plus qu'il ne peut se voir dicter d'avance (ou au contraire interdire) aucune sorte de matériau ou de combinaison de matériaux. Mais si la variété imprévisible de ces derniers, tout comme celle des secteurs de l'activité humaine où elle trouve à s'exercer, tendent vers son indéfinition par excès d'hétérogénéité, l'activité du dessin désigne pourtant, $d u$ point de vue de son processus, une zone spécifique : celle où les choses parviennent au langage, par le truchement d'un geste d'écriture qui prolonge leur perception et dont elles sont le premier moteur.

\section{Réalité des relations et langue des objets}

La conversation à propos du dessin que Beuys a conduite en 1979 avec Heiner Bastian et Jeannot Simmen peut servir à éclaircir ces points. Beuys y énonce en effet clairement ce qui constitue à proprement parler la condition d'exercice du dessin, à savoir le caractère réel des relations entre les choses:

"Je crois aussi que les choses ont entre elles et les unes avec les autres des liaisons vraiment tout à fait réelles, et qu'elles sont en conversation les unes avec les autres, aussi hétérogènes que soient les choses, et souvent les formes et ce à quoi elles s'adressent. ${ }^{7}$

La possibilité du dessin prend naissance dans l'existence réelle de la relation dont il assume la représentation. Qu'il dénote une fleur ou une idée, il s'intéresse toujours à la réalité de son objet, réalité qui inclut le réseau de circonstances où celuici apparaît et avec lequel il est en interaction. Bien plus, en tant que réalité, l'objet lui-même est déjà réseau de circonstances et diagramme de forces. Pour être doté d'une stabilité relative, il n'en continue pas moins d'entretenir des relations avec

5. Ibid. : 50-51 (mes italiques).

6. On citera ici la définition des épiphanies attribuée par Joyce à son héros Stephen dans Stephen le héros, la première version du Portrait de l'artiste en jeune homme: «Il entendait par épiphanie une manifestation spirituelle soudaine, qu'il s'agisse de la vulgarité du discours ou du geste, ou d'une phase mémorable de l'esprit lui-même. Il croyait qu'il appartenait à l'homme de lettres d'enregistrer ces épiphanies avec un soin extrême, voyant qu'elles sont elles-mêmes les plus délicats et évanescents des moments " . Voir James Joyce, Euvres, trad. modifiée par l'auteur. Paris, Gallimard, 1982, I : 512

7. Voir Joseph Beuys, Zeichnungen, Munich, Prestel, 1979: 40 (mes italiques). 


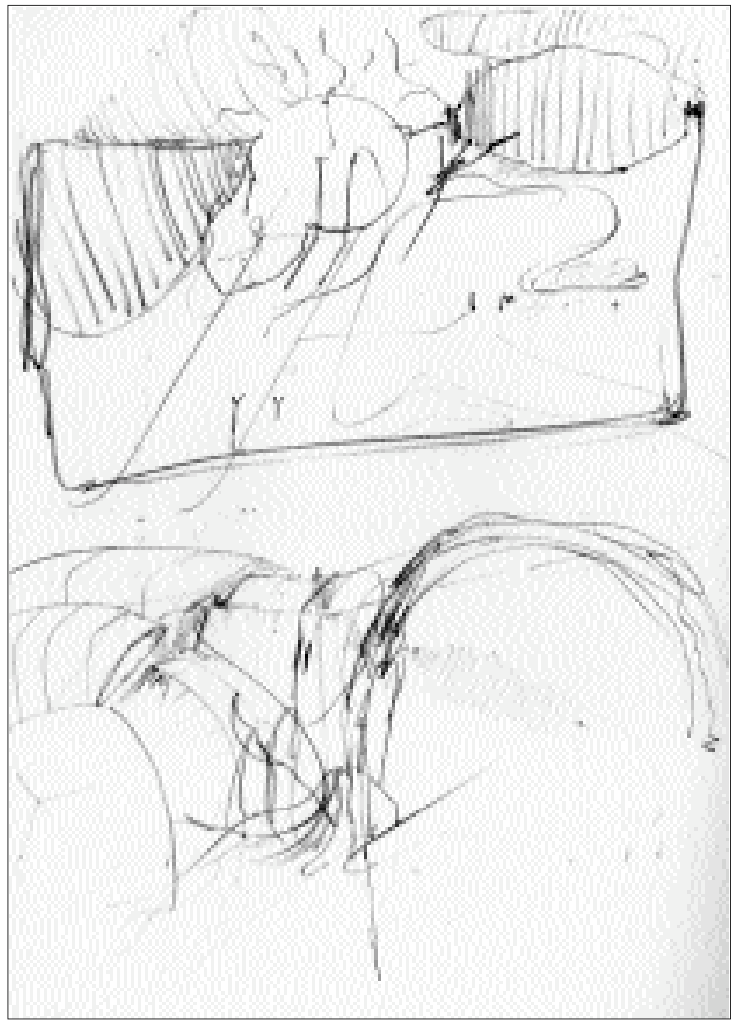

Fig. 2 Wärmeplastik im Gebirge, 1956, dessin au crayon, 21 × 29,6 (Joseph Beuys, Natur..., op. cit., I99I: pl. 49)

Fig. 3 Diagramm-Zeichnung, 1972 craie sur ardoise, $200 \times 150 \mathrm{~cm}$ (Joseph Beuys, Natur..., op. cit., 1991: pl. 207)

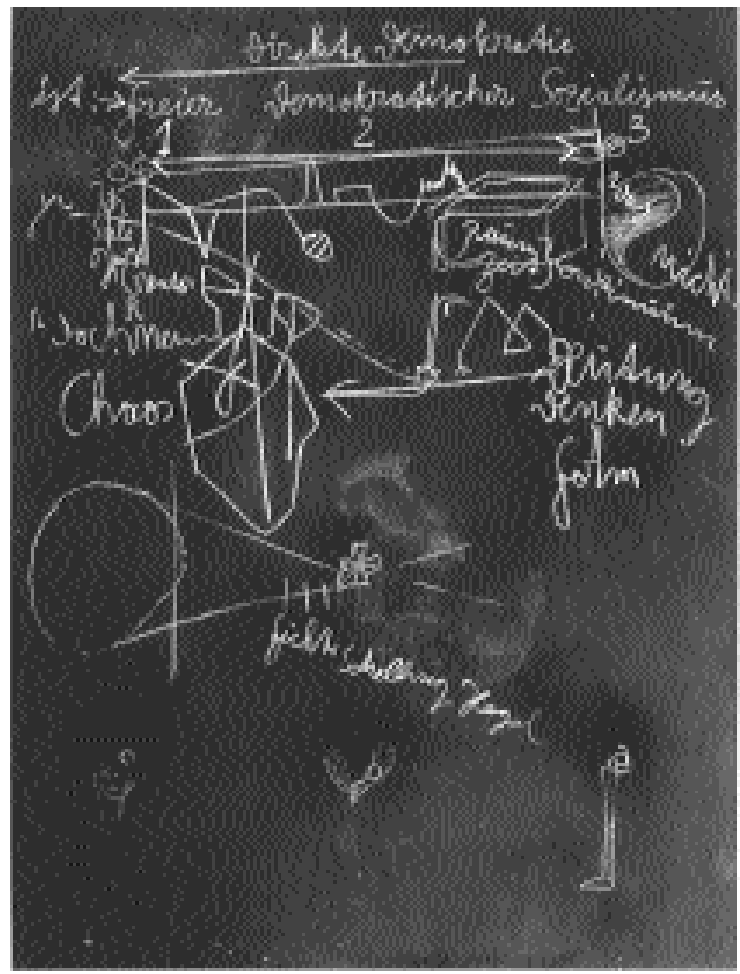


le reste des forces objectifiées ou fluides qui l'entourent, y compris celles de l'individu voulant dessiner. Ce sont ces diagrammes de forces que le dessin a pour tâche d'enregistrer et de fixer, faisant passer la réalité de leur existence dans une forme entièrement visible.

Ce trait explique la concomitance de deux affirmations qui pourraient aisément passer pour contradictoires. La première est celle de la nécessité, pour démarrer le processus, qu'un objet singulier se déclare :

«Je ne m’assois (pour dessiner) que si une nécessité existe, si une chose quelconque se déclare. Si rien ne se déclare, alors je ne dessine pas. C'est-à-dire si un objet qui veut se représenter s'affirme quelque part, s'il dit : je veux, je dois être représenté maintenant, parce que c'est nécessaire que je sois représenté, alors c'est là que je me mets à dessiner. " ${ }^{8}$

La seconde est celle de la présence, au départ de ce même processus, d'une multiplicité à organiser :

"C'est un processus très intime, qu'il est très difficile d'exprimer avec des mots. Ainsi il faut que soient disponibles des complexes entiers et des constellations. Il faut qu'il $\mathrm{y}$ ait une très complexe sphère d'intérêts, et cette sphère ne prend pas forme bien sûr seulement par passivité, mais par le fait de mettre une logique réelle dans l'ensemble des circonstances de sa vie et de vraiment faire le sale boulot. Donc faire un travail qui en apparence se situe ailleurs, dans une tout autre sphère que par exemple le dessin. „"

Dans ces propos, Beuys pose plusieurs affirmations. La première est que chaque chose singulière existe comme multiplicité, tout comme les relations, elles aussi singulières, qu'elle entretient avec ce qui l'entoure. Si elles different en temps, lieu, forme et disposition, elles sont, l'une comme les autres, de même niveau, car produites par un jeu de forces réelles. Cela explique deux autres traits. Beuys semble en effet dans ses propos attribuer aux choses, tout comme bien des «primitifs ", une capacité à se déclarer indépendantes de toute décision consciente du dessinateur. Les objets affirment un "droit à la représentation", que leur "volonté" impose ou tente d'imposer à la personne qui dessine. Il y a là une manière de personnification qui apparaît facilement comme le simple déni du processus par lequel un dessinateur décide d'appliquer son attention et son savoir-faire à tel objet, plutôt qu'à tel autre. Beuys revendique pourtant une part active pour le dessinateur, par delà la passivité que semble impliquer l'acceptation des «volontés" de l'objet comme point de départ de l'impulsion au dessin ${ }^{10}$. Le paradoxe est alors que cette activité, ce travail, n’a pas pour lieu spécifique le dessin. Il se détermine plutôt, en amont, comme nécessité de réorganiser «l'ensemble des circonstances de la vie » du dessinateur, préalablement à toute considération ou du résultat fini et de son activité.

8. Ibid. : 38 .

9. Ibid.

10. Bastian : "Mais tu dois bien pourtant [pour dessiner] te décider pour un objet déterminé ? " Beuys : «Non, l'objet doit d'abord s'être déclaré » (ibid.). 
La "volonté » de telle ou telle chose de parvenir à la représentation, bien qu'elle soit à la source de l'impulsion à dessiner, n'a en effet jamais pour résultat premier un dessin. Elle est plutôt l'événement par lequel une chose (un réseau de relations) s'impose à l'attention, déclare son caractère réel, rendant du même coup visibles les complexes et constellations qui lui donnent forme. La force par laquelle cette chose s'impose à la volonté déclenche alors chez le dessinateur un contre-travail de mobilisation de forces, et de mise en forme de la "logique réelle», sans lequel le processus du dessin ne peut aboutir. Aussi dans chaque tentative de dessiner, achevée ou non, est-ce bien la confrontation avec les choses qui règne au premier chef, le dessinateur étant lui-même une des choses réelles qui se confrontent les unes aux autres, lui-même un contenu du monde ${ }^{11}$.

C'est à ce niveau que prend sens la personnalisation des objets et des relations effectuée par le discours de Beuys. "Personnes composées de personnes" au même titre que les aventures et chapitres d'Ulysse pour leur auteur ${ }^{12}$, les uns et les autres ne constituent pas pour lui des entités séparées, simples et autonomes, mais toujours «des complexes entiers et des constellations» dont les apparitions forment réseau. Si ces complexes sont perçus comme ayant le pouvoir de «se déclarer ", de "vouloir ", ou même d'entretenir des "conversations" les uns avec les autres, c'est en tant qu'ils imposent la force de leur individualité à d'autres "personnes» réelles singulières, elles aussi capables de volonté. Le mouvement de personnification des choses se compose alors en retour avec un contre-mouvement : celui par lequel le dessinateur potentiel se découvre comme constellation ou complexe de relations exerçant une force ou une résistance à l'égard d'autres individus réels ; celui par lequel il se découvre comme croisement individué d'expériences qui, comme les autres, réclament de "se représenter " à l'aide de diagrammes et d'énoncés spécifiques.

L'ambition pour le dessin de représenter quelque chose de réel passe alors de façon tout à fait fondamentale - avant toute problématique de réalisation mimétique ou stylistique - à la fois par le respect de la perception initiale de l'objet comme s'imposant de lui-même à l'attention, et par un travail portant sur la logique qui informe le matériau d'expérience propre à l'individu dessinant. C'est bien en effet parce que les relations se déploient au niveau de l'existence réelle des

11. Sur le rôle que joue cette imposition du réel dans le déclenchement de l'impulsion à dessiner, voir l'entretien de Beuys avec Hans van der Grinten ("Dialogue», in Max Reithmann, Joseph Beuys..., op. cit., 1994 : 19) : "Quand on entre dans les choses complexes, dans les contenus du monde, on aimerait certainement les reformuler, ou du moins formuler cette confrontation même. Celle-ci peut avoir à chaque fois plusieurs origines. Elle peut être de l'ordre de la pensée, du sentiment, de la sensation, de l'expérience vécue. Elle peut enfin avoir sa source dans les incitations de la volonté. " (mes italiques.)

12. Voir la lettre de James Joyce à Carlo Linati concernant l'écriture d'Ulysse: il y décrit son œuvre comme « l'épopée de deux races [Israël et Irlande] en même temps que le cycle du corps humain et aussi la petite histoire d'une journée [vie]. [...] C'est aussi une sorte d'encyclopédie. Mon intention n'est pas seulement de rendre le mythe sub specie temporis nostri, mais de permettre que chaque aventure, c'est-àdire chaque heure, chaque organe, chaque art connecté et identifié dans le schéma somatique du tout) conditionne, et même crée, sa propre technique. Chaque aventure est pour ainsi dire une personne, bien que composée de personnes - comme le raconte l'Aquinate des armées angéliques » (James Joyce, lettre du 21 septembre 1920 à Carlo Linati, Euvres, Paris, Gallimard, 1995, II : 910). 
objets du monde que leur interaction réciproque se constitue en un langage potentiel : en une "conversation ». Et ici se marque la spécificité du rôle du dessin en tant que lieu du passage vers la langue.

\section{Une langue d'avant et d'après les symboles}

Beuys insiste à maintes reprises sur l'importance du dessin comme lieu d'accès des choses au langage, et cela alors qu'il tend à décrire ces mêmes choses, en tant que complexes de relations, comme déjà engagées, avant toute intervention humaine, dans quelque chose qui vaut comme une conversation. C'est que le lieu du dessin est double. On l'a dit : "rien de plus élémentaire que le dessin ». Il inclut tout geste de la main qui dénote ou évoque quelque chose, qui rapproche deux objets quelconques, aussi hétérogènes soient-ils, pourvu que ce geste induise une trace matérielle. Tout dessin est une icône, dans le sens que donne au mot le philosophe américain Charles S. Peirce: «N'importe quoi, qualité, individu existant ou loi, est l'icône de quelque chose, pourvu qu'il ressemble à cette chose et soit utilisé comme signe de cette chose. ${ }^{13}$ Cette définition entre en effet en résonance immédiate avec le propos de Beuys. Dessiner, c'est pénétrer, avec n'importe quel instrument ou matériau, dans la dimension iconique ; non pas, comme on pourrait le croire, parce que de nombreux dessins présentent des ressemblances optiques immédiatement perceptibles comme telles par les yeux occidentaux qui les regardent, car inscrites dans des codes connus, mais parce que toute trace gestuelle ordonne des ressemblances virtuelles, y compris avec des choses inconnues ou invisibles. Elle est le lieu matériel de qualités qui, existant là pour elles-mêmes, n'en sont pas moins susceptibles d'être transportées d'un objet à un autre, au gré des circonstances et des associations.

Dessiner, c'est donc fixer une qualité, ou plutôt une constellation de qualités. En tant qu'elles prennent corps, ces qualités acquièrent une consistance réelle. Mais en tant que qualités, elles continuent d'exhiber des possibilités, et font du dessin beaucoup plus qu'une ressemblance assignable selon des critères prédéterminés : le lieu de ressemblances à venir et à inventer.

En ce sens, on peut bien parler, à propos de ce qui déclenche l'impulsion vers le dessin, de déclaration d'un objet, alors même que cette déclaration précède toute énonciation discursive d'ordre symbolique. Des qualités s'avancent, disposées en une certaine constellation ou forme. Elles entrent en relation avec des instances de même qualité appartenant à d'autres objets. Elles organisent une circulation des ressemblances et différences qui forme un "langage d'images" (Bildersprache) ${ }^{14} . \mathrm{Qu}^{\prime}$ " un objet se déclare" signifie alors qu'une ressemblance potentielle se constitue dans les choses perçues. Pour acquérir consistance réelle, cette ressemblance devra en passer par l'opération pratique du dessin.

13. Charles Sanders Peirce, Écrits sur le signe, choix et traduction de Gérard Deledalle, Paris, Le Seuil, $1978: 140$.

14. Voir l'entretien avec Hans van der Grinten, "Dialogue ", in Max Reithmann, Joseph Beuys..., op. cit., 1994 : 21. L'expression souligne la dimension iconique du langage dessiné. 
La déclaration des choses, que Beuys tend à présenter comme antérieure à sa projection matérielle, n'existe en effet pas séparément de cette opération. C'est seulement après avoir effectué le geste qui en délivre et fixe la trace qu'on pourra affirmer qu'elle préexistait dans les choses. On voit ici poindre le deuxième caractère du dessin : parce qu'il est trace, empreinte, il produit la ressemblance sous la forme de quelque chose qui était déjà là, et qu'il semble se contenter de re-présenter a posteriori. Aussi, si le dessin est icône, au sens précédemment défini, il est aussi indice, pour continuer d'exploiter la tripartition du signe établie par Peirce en icône, indice et symbole ${ }^{15}$.

Tout signe dessiné est à la fois icône et indice : icône des constellations qưil évoque et dont il présente le diagramme ; indice des gestes qui l'ont bâti, et dont son matériau conserve l'empreinte. Le lieu commun de ces deux caractères est la matérialité de l'objet. L'attention portée à ces deux aspects explique l'importance qu'a pour Beuys l'invention et la caractérisation précise des matériaux qu'il utilise. Leur variété, leurs propriétés physiques (ou qualités), enfin les objets et les activités souvent extra-artistiques auxquels ils renvoient font partie de l'information diffusée :

"Je veux dire, il n'y a pour l'homme, tant qu'il est homme, absolument aucune autre possibilité de s'exprimer par rapport à autrui si ce n'est par un processus de substances. Même quand je parle, j'ai besoin de ma glotte, de mes os, d'ondes sonores, j'ai besoin par exemple de la substance de l'air : vous avez absolument besoin d'une membrane dans l'oreille sinon vous n'entendriez pas du tout ce que je dis. Il n'y a pas d'autre possibilité de transmission si ce n'est par une empreinte qu'on laisse dans un certain matériau. [...] Pour obtenir de l'information, il faut toujours saisir des relations de substances, en un sens très général. C'est-à-dire que l'échange d'informations entre humains n'est pas pensable sans substances. $»^{16}$

Le terme de substance ici utilisé pour désigner la matérialité de l'œuvre rend compte de cette double problématique. La substance est au sens prop re ce qui se tient au-dessous: le lieu-support des qualités, et à ce titre celui des ressemblances v i rtuelles. Mais elle est aussi ce qui ne se résout pas dans la pure surface d'une visibilité présente, et ce qui relie cette visibilité, d'une part, aux constellations de fo rces, invisibles mais réelles, qui la soutiennent, dautre part, au devenir de ces fo rces, dont le dessin fixe un moment, fugitif, mais affecté par le passé qui est venu s'y inscrire :

«La substance devient naturellement importante [...] pour par exemple s'éloigner du domaine rétinien, c'est-à-dire de ce type de transmission dans le pur domaine formel, pour sentir le contexte total des forces. Et les contextes de forces se déroulent dans des contextes de substances, ce ne sont pas de purs facteurs rationnels, analytiques, où l'on interprète l'information en la décomposant analytiquement, par exemple selon la

15. Un indice est « un signe qui renvoie à l'objet qu'il dénote parce qu'il est réellement affecté par cet objet. [...] Dans la mesure où l'indice est affecté par l'objet, il a nécessairement quelque qualité en commun avec l'objet, et c'est eu égard à ces qualités qu'il peut avoir en commun avec l'objet qu'il renvoie à cet objet ", C. S. Peirce, Écrits sur le signe..., op. cit., $1978: 150$.

16. Joseph Beuys-Volker Harlan, Qu'est-ce que l'art?, op. cit .. 1992 : 112 (mes italiques). 
méthode de la logique; au contraire la logique se prolonge, il faut pour cela des organes qui relèvent de l'intuition, de l'inspiration et de l'imagination, sinon on ne ressent rien. ${ }^{17}$

Ce commentaire de Beuys sur le caractère d'opérateur irrationnel propre aux substances - c'est-à-dire au matériau de l'inscription du geste - amène à considérer le dernier aspect du dessin: la résistance que cette combinaison d'indice et d'icône oppose à toute résorption achevée dans la symbolisation. Le dessin, s'il est toujours à la fois icône et indice, ne devient jamais pur symbole ${ }^{18}$. Il est et demeure antérieur au passage dans le symbolique qu'il annonce et rend possible. En tant que tel, il incarne alors une dimension archaïque du langage : le moment où l'articulation des relations apparaît telle quelle dans les matériaux et la réalité du monde, mais où elle présente une forme qui n'est pas assujettie à des lois générales. Le caractère inchoatif du dessin, déjà signalé à plusieurs reprises, n'est donc pas lié à l'émergence d'un objet perçu dans son essence muette, prélangagière. Il l'est à l'apparition de la relation sous forme d'une singularité saisie comme telle, singularité qu'il est impossible de porter tout entière vers le symbole sans estomper, voire détruire, son caractère propre de réalité hic et nunc.

\section{Une langue d'après les symboles}

Cette capacité à saisir la relation au moment où elle s'invente et se découvre donne son prix au dessin. Mais ce trait, qui fait de lui le lieu privilégié de l'élaboration des pensées, n'en fait pas pour autant un commencement accessible de manière immédiate. Si le dessin est conceptuellement le lieu dinvention de la langue, avant sa solidification achevée en symboles (les mots), la perception de cette dimension se heurteàl'histoire moderne des fonctions assignées au dessin et à l'écrituredans les sociétés occidentales. Cette histoire, on l'a vu, repose sur le privilège accordé à une dimension iconique-diagrammatique détachée de la dimension indicielle, cette dernière étant réduite à la marque stylistique d'un individu a rtiste. À la prédominance de la relation icônelindice, qui faisait la valeur heuristique du dessin, succède la domination de la relation icônelsymbole. Celle-ci met l'accent sur une ressemblance dont la performance obéit à des lois générales préétablies. La fonction symbolique du dessin prend alors le dessus sur son caractère d'invention gestuelle de la pensée; d'une part, avec le développement du dessin technique et de schémas scientifiques mathématico-physiques; dautre part, avec le triomphe, à l'âge de la représentation, d'une mimésis artistique fondée sur le rendu précisément réglé des transformations morphologiques qu’opèrent sur les objets les variations de distance par rapport à l'œeil d'un observateur.

Dans cette configuration, la dimension indicielle du dessin ne disparaît pas (comment le pourrait-elle?), mais elle se trouve presque entière cantonnée dans

17. Ibid. : 113-114.

18. Un symbole est " un signe qui renvoie à l'objet qu'il dénote en vertu d'une loi, d'ordinaire une association d'idées générales, qui détermine l'interprétation du symbole par référence à cet objet ", C. S. Peirce, Écrits sur le signe..., op. cit., 1978 : 140-141. 
le geste et dans ses accidents, conçus comme écarts par rapport à un idéal d'imitation porté jusqu'au statut de loi symbolique. Cet écart, réduit à son minimum voire nié dans le dessin scientifique, trouve alors son lieu propre dans le domaine artistique, avec le développement, dans la deuxième moitié du XVIII siècle puis avec le romantisme, d'une systématisation de l'accident et des micro-événements qui reconfigurent - toujours dans le cadre de la scission déjà opérée - la relation icônelindice ${ }^{19}$.

Il ne s'agit pourtant pas pour Beuys de poursuivre la réhabilitation romantique de l'écart, contre une ligne rationalisante icône/symbole qu'incarneraient de concert le dessin scientifique et l'imitation classique. D'une part, il ne peut être question de condamner le développement du premier, dont la puissance d'invention est une extension très réelle de la créativité humaine, et une authentique métamorphose de la conscience. D'autre part, la posture romantique de l'écart, bien qu'elle remette en question la relation de surface entre icône et symbole au profit de la relation de profondeur icône/indice, témoigne encore de sa dépendance à l'égard des systèmes classique et scientifique qu'elle subvertit.

C'est dans ce contexte qu'il faut entendre le recours de Beuys à des modèles archaïques et/ou non occidentaux, exempts de la scission historique entre dessin scientifique et dessin artistique. Le réélargissement qu'établissent les allusions aux peintures rupestres ou de sable permet la restauration d'un plan élémentaire : à l'écart des différenciations qui ont marqué, mais aussi limité, son histoire moderne, l'activité du dessin y redevient pensable en général comme l'effectuation de traces qui font signe non pas parce qu'elles appartiennent à des registres encodés par avance, mais parce qu'elles savent produire, à partir d'un petit nombre de cellules gestuelles différenciées (par exemple les croix, cercles, zigzags mentionnés par Beuys) des ressemblances graphiques en nombre virtuellement infini. La revendication beuysienne de modes archaïques et exotiques (qui plus est associés à des formes de réalisation souvent éphémères) n'est donc pas celle d'une régression historique, visant à faire table rase du passé récent et du développement de la rationalité. Elle est la revendication pour le dessin d'une position structurellement archaïque : celle de commencement, d'ébauche des processus de pensée, en tant que facteur et moment du passage vers la langue et vers l'écriture.

Le statut que Beuys accorde à l'écriture elle-même aide à clarifier ce dernier point. Dans la mesure où elle reste associée à la mise en exercice de la langue, l'écriture est porteuse de symboles: les mots et les phrases que commandent lois syntaxiques et associations d'idées générales. Pourtant, Beuys fait du passage de la langue de la parole vers l'écriture le premier moment de son élargissement et de sa refluidification dans le dessin. L'inscription des concepts et des énoncés dans l'écriture, loin de les rigidifier, est l'occasion de leur remise en jeu en tant que traces événementielles réelles, du même coup susceptibles de se voir défaites, puis reconstruites, de manière neuve :

19. Voir, à ce sujet, le livre fondamental de Jean-Claude Lebensztejn, L’Art de la tache, Montélimar, Éditions du Limon, 1990. 
"C'est sûrement une pulsion générale des hommes, que de consigner, pour ainsi dire, les choses dans des produits. Je pourrais me figurer que beaucoup s'en tiennent, pour cette production, au moyen d'expression du langage, c'est-à-dire en restent à la parole. D'autres feraient peut-être de cette parole quelque chose de tout à fait matériel, un objet, en mettant le langage par écrit. Et ici on en est justement au point précis où, simplement par l'écriture sous forme de lettres, puis de concepts, puis de phrases, on élargit la saisie. C'est dans cette position d'élargissement du domaine du langage quion cherchera mon impulsion de base concernant l'activité du dessin. ${ }^{20}$

On le voit, la revendication d'une position archaïque pour le dessin est en fait celle du réélargissement, à partir du présent actuel hyperrationalisé, d'un mode d'intervention auquel aucune loi, pas même celles qui régissent l'expression du langage ordinaire, ne doit prescrire de limites a priori. Mais le dessin, "langue" dont le processus prend corps avant la constitution de symboles achevés, n'existe alors aujourd'hui, du point de vue de son accès, que comme la possibilité d'une langue d'après les symboles. Travaillant avec eux, il doit les casser pour les refluidifier, et ainsi parvenir à ce qui est sa mission première, en même temps que le plus petit dénominateur commun des multiples activités nommées dessin : une institution désymbolisée du sens. D'où la nécessaire vigilance que réclame le redéploiement de la dimension présymbolique, inchoative. D'où également le caractère archéologique de cette dernière démarche :

"J'essaie de garder le caractère de langage [du dessin] dans la plus grande fluidité et mobilité possibles, pour dépasser l'usurpation du langage par le développement culturel et la rationalité. Je veux atteindre une compréhension élargie qui permette d'exprimer des choses, qui mène à des domaines plus profonds. C'est cet élargissement de la langue qui me fait m'intéresser au dessin. $»^{21}$

La position "archaïque " du dessin se révèle alors ne pas tenir à une antériorité historique primitive, au regard d'une pensée symbolique entièrement identifiée aux solidifications des langues modernes et à la pensée scientifique dont elles ont permis le développement. Elle tient plutôt à une position structurelle dans le processus de constitution de l'activité de pensée (et cela y compris dans notre présent) : celle d'un commencement. Elle tient aussi à un travail spécifique: instituer les plans à partir desquels le processus de symbolisation prend son essor, pour se cristalliser en un langage constitué ${ }^{22}$.

20. Voir « Dialogue », op. cit., 1994 : 20 (traduction modifiée, J.-P. A., mes italiques).

21. Ibid. : 20-21 (traduction modifiée, J.-P. A.).

22. Les aspects "primitivistes " d'une partie de la production dessinée de Beuys peuvent de ce point de vue prêter à confusion. Ils semblent avaliser une position régressive par rapport à l'histoire moderne - et moderniste - du dessin. Mais il s'agit pour l'artiste d'une réouverture vers des possibles oubliés, non pas d'une nostalgie prenant pour objet une origine mythique : "On me reproche souvent de retourner en arrière sous prétexte que je fais remarquer que, dans le passé, tous ces principes étaient lisibles. En évoquant, par exemple, d'une manière purement formelle, certains exemples chamanistes, je ne veux que signaler l'existence de ces forces. Mais je n'ai aucune intention, comme d'aucuns le prétendent, de retourner dans le sein maternel ou dans ma baignoire d'enfant. Tous ces exemples ne me servent qu'à démontrer dans quel sens nous devrons nous diriger à l'avenir. ce qui devra être encouragé et ce qu'il faudra empêcher de croître par tous les moyens. " (Irmeline Lebeer, "Entretien avec Joseph Beuys... ", op. cit., 1980 : 181). 
Réhabiliter la valeur du dessin, et surtout reconnaître la spécificité de sa fonction dans les processus de pensée, c'est alors ouvrir la voie à une analyse de toutes 142 les traces appartenant à des systèmes non entièrement formalisés, exempte des préjugés les plus tenaces qui la menacent. Le premier est un primitivisme toujours empreint de l'idée de progrès et des condescendances de la rationalité occidentale : confondant antériorité historique et position archaïque, il est aveugle devant ce qui, dans le présent en travail, continue de relever de commencements de pensée. Le second, plus sophistiqué et aujourd'hui plus répandu, consiste, sous le prétexte louable de mieux leur rendre leur dignité, à tenter de présenter sous forme de langages entièrement symboliques et formalisés des traces dont la nature est au contraire de refuser de s'intégrer à des systèmes formels clos, et de maintenir ouvertes diverses possibilités de cristallisation pour des pensées en devenir.

Les propos - et l'œuvre - de Beuys aident à la prise en considération de cette dimension propre du dessin, un dessin dont le domaine cesse d'être abusivement restreint aux Beaux-Arts, voire à l'art issu du dépassement des Beaux-Arts. Il embrasse le terrain beaucoup plus vaste, mais non mutilé conceptuellement, de l'ensemble des traces et marques au moyen desquelles les hommes témoignent les uns envers les autres du cours de leur activité de pensée, avant sa solidification achevée en symboles, mais déjà sous une forme capable d'instituer de possibles lieux communs du sens.

MOTS CLÉS/KEYWORDS: dessin/drawing - penséelthought - primitivisme/primitivism - art Joseph Beuys.

RÉSUMÉ/ABSTRACT

Jean-Philippe Antoine, De l'archaïque au commencement: la pensée du dessin chez Joseph Beuys - "Penser, c'est dessiner", déclare l'artiste Joseph Beuys. Élargie à tout geste de désignation se cristallisant dans une trace, la pratique du dessin échappe aux catégorisations partielles (Beaux-Arts, dessin scientifique et technique, style) qui en ont, depuis plusieurs siècles, restreint l'usage et la portée. Elle retrouve ainsi le rôle de commencement de pensée et de "langue d'avant les symboles" qui est le sien dans la plupart des cultures.
Jean-Philippe Antoine, From the Archaic to the Beginning:Joseph Beuy's Thoughts on Drawing. The artist Joseph Beuy has said, "To think is to draw ». Broadened to cover any gesture of po rtrayal that crystallizes in a line, the practice of drawing escapes from partial categorizations (Fine Arts, draftsmanship, style), which have, for several centuries now, restrained its use and scope. It thus rediscovers the role of the beginning of thought and of the "language before symbols » which belongs to it in most cultures. 